\title{
Is it Necessary to Perform Drug-induced Sleep Endoscopy in Severe Obstructive Sleep Apnea?
}

\author{
Shrinath Dayananda Kamath Patla ${ }^{1}$, Remya Koikkara²
}

\begin{abstract}
Aim: To study the role of drug-induced sleep endoscopy (DISE) and Muller's maneuver in diagnosing obstructive sleep apnea (OSA). Materials and methods: This was a comparative study done on 30 patients above the age of 18 years with OSA. All the study subjects underwent Muller's maneuver and DISE. In both endoscopic procedures, the degree and type of airway collapse noted at the retropalatal and retrolingual levels.

Result: Our study revealed similar findings with regard to type and degree of airway collapse at the retropalatal and retrolingual levels with both Muller's maneuver and DISE.

Conclusion: The type and degree of upper airway collapse at the retropalatal and retrolingual levels in DISE were similar to that of Muller's maneuver in severe OSA. Hence, DISE is not necessarily a diagnostic tool in case of severe OSA.

Keywords: DISE, Muller's maneuver, OSA, Polysomnography.

Clinical Rhinology An International Journal (2019): 10.5005/jp-journals-10013-1350
\end{abstract}

\section{INTRODUCTION}

In earlier days in patients with obstructive sleep apnea (OSA), upper airway examination was performed with the patient awake. In the last two decades, drug-induced sleep endoscopy (DISE) has been used for tridimensional evaluation of the upper airway following pharmacologically induced sleep.

Many studies have demonstrated that DISE is a simple, safe, and cost-effective diagnostic approach to OSA. ${ }^{2}$ But, there are only few studies comparing DISE and awake upper airway examination with regard to its usefulness for appropriate treatment modalities. In this study, we compared the effectiveness of DISE and awake Muller's maneuver in identifying the site and pattern of airway collapse in OSA patients.

\section{Materials and Methods}

A prospective comparative study was conducted in the Department of ENT, KS Hegde Medical Academy, Mangaluru, from November 2016 to September 2018 after obtaining the institutional ethical committee clearance. Thirty patients above the age of 18 years with severe OSA diagnosed by polysomnography and without any previous surgical therapies for OSA were included in the study.

All the patients diagnosed with OSA in polysomnography were subjected to nasal endoscopic airway evaluation with Muller's maneuver in awake state and followed by DISE. The type and degree of airway collapse at the retropalatal and retrolingual levels were noted. The findings of the two approaches were compared in all study patients.

\section{Muller's Maneuver}

Muller's maneuver was performed by a single surgeon on all the study subjects using a fiber optic endoscope in awake state. All study subjects were taught on how to carry out the maneuver. Nasal decongestion and topical anesthesia were achieved with $4 \%$ lignocaine and adrenaline. In sitting position, a flexible fiber-optic endoscope (Karl Strorz fiberscope) was passed through one nostril
1,2Department of ENT, KS Hegde Medical Academy, Mangaluru, Karnataka, India

Corresponding Author: Remya Koikkara, Department of ENT, KS Hegde Medical Academy, Mangaluru, Karnataka, India, Phone: +91 9035922369, e-mail: rkoikkara@gmail.com

How to cite this article: Patla SDK, Koikkara R. Is it Necessary to Perform Drug-induced Sleep Endoscopy in Severe Obstructive Sleep Apnea? Clin Rhinol An Int J 2019;12(1):3-5.

Source of support: Nil

Conflict of interest: None

to visualize the nasopharynx and entire upper airway. Subsequently, the patient was asked to perform Muller's maneuver by maintaining maximal inspiration with closed glottis against closed oral and nasal airways. The same maneuver was performed when the endoscope was positioned above the retropalatal and retrolingual levels. The degree of airway collapse was evaluated as a percentage change in the cross-sectional airway and divided into four groups of $<25 \%$, $25-50 \%, 50-75 \%$, and $>75 \%$ at each site. The type of collapse was noted as longitudinal, circumferential, or anteroposterior.

\section{Drug-induced Sleep Endoscopy}

Sleep endoscopy was done in the operating room by a single surgeon in all subjects. In a controlled monitored setting, IV infusion of dexmedetomidine $1 \mu \mathrm{g} / \mathrm{kg}$ over 20 minutes was followed by $0.3 \mu \mathrm{g} / \mathrm{kg} / \mathrm{hour}$ infusion given. Hemodynamic and respiratory parameters are observed. Once sedation is achieved (after 15 minutes of infusion), a flexible fiber optic endoscope (Karl Storz fiberscope) was passed through one nostril, and the nasopharynx and entire upper airway were inspected. Dynamic collapse of the upper airway was noted and described in terms of percentage. The cross-sectional area change based on anteroposterior and lateral axes of collapse was noted. The estimated degree of airway collapse at each level was noted as $>25 \%, 25-50 \%, 50-75 \%$, and $>75 \%$. 
The type of collapse as longitudinal, circumferential, and anteroposterior was also noted.

The collected information was analyzed by frequency and percentage. The agreement between the two categories was analyzed by Kappa statistics. The analysis was performed using Statistical Package for the Social Sciences-17 (SPSS-17). The level of significance of the study was 0.05 . The analysis was performed using the Statistical Package for the Social Sciences (SPSS) software-17. The $p$ value of 0.05 was considered as significant. The calculated $k$ values are interpreted as follows: $<0.2$ (poor agreement), $0.2-0.4$ (fair agreement), 0.4-0.6 (moderate agreement), 0.6-0.8 (good agreement), and 0.8-1.0 (very good agreement).

\section{Results}

The present study was done on 30 patients diagnosed with OSA by polysomnography and compared their airway collapse during endoscopic evaluation of upper airway with Muller's maneuver in awake state and followed with DISE.

The mean age of the study subjects was 39.6 years. Most cases of OSA patients were in the age group of $31-40$ (40.0\%) and $41-50$ years $(26.7 \%)$. In our study, males had preponderance over females (93.3\%). Of the 30 cases evaluated for OSA, 14 cases (46.7\%) were obese. Most of our patients had an AHI of 30-60. None of our patients had $<30$. All patients had severe OSA $(>30)$.

With regard to the degree of collapse at the retropalatal level, $50-75 \%$ collapse was seen in $16.7 \%$ and $10.0 \%$ in Muller's maneuver and DISE, respectively, and more than $75 \%$ collapse was seen in $83.35 \%$ and $90.0 \%$ in Muller's maneuver and DISE, respectively (Table 1). The $p$ value was 0.014 which was statistically significant. The calculated $\mathrm{k}$ value was 0.429 , which signifies there is a moderate agreement between both methods showing similar degree of collapse at the retropalatal level.

With regard to the degree of collapse at the retrolingual level, $50-75 \%$ collapse was seen in $13.3 \%$ and $3.3 \%$ in Muller's maneuver and DISE, respectively, and more than $75 \%$ collapse was seen in 83.35\% and $90.0 \%$ in Muller's maneuver and DISE, respectively. The $p$ value was 0.010 which was statistically significant. The calculated $\mathrm{K}$ value was 0.366 with both the methods fairly agreeing with each other (Table 2).

At the retropalatal level, majority of patients showed circumferential collapse (53.3\% and 63.3\%) in Muller's maneuver and DISE, respectively (Table 3 ). The $p$ value was 0.001 and was

Table 1: Comparison of degree of airway collapse at retropalatal level in Muller's maneuver and drug-induced sleep endoscopy (DISE)

\begin{tabular}{lcc}
\hline $\begin{array}{l}\text { Percentage degree } \\
\text { of airway collapse }\end{array}$ & Muller's maneuver & $\begin{array}{l}\text { Drug-induced sleep } \\
\text { endoscopy }\end{array}$ \\
\hline $50-75 \%$ & $5(16.7 \%)$ & $3(10.0 \%)$ \\
$>75 \%$ & $25(83.3 \%)$ & $27(90.0 \%)$ \\
Total & $30(100.0 \%)$ & $30(100.0 \%)$ \\
\hline
\end{tabular}

Table 2: Comparison of degree of airway collapse at retrolingual level in Muller's maneuver and drug-induced sleep endoscopy (DISE)

\begin{tabular}{lcc}
\hline $\begin{array}{l}\text { Degree of airway } \\
\text { collapse (\%) }\end{array}$ & Muller's maneuver & $\begin{array}{l}\text { Drug-induced sleep } \\
\text { endoscopy }\end{array}$ \\
\hline $50-75 \%$ & $4(13.3 \%)$ & $1(3.3 \%)$ \\
$>75 \%$ & $26(86.7 \%)$ & $29(96.7 \%)$ \\
Total & $30(100.0 \%)$ & $30(100.0 \%)$ \\
\hline
\end{tabular}

statistically significant. The calculated $\mathrm{k}$ value was 0.467 . Both the methods agreeing moderately with each other.

At the retrolingual level, there was a similar pattern of collapse seen in Muller's maneuver and DISE. $73.3 \%$ showed circumferential collapse in both the procedures. $75.0 \%$ showed a similar pattern of collapse (Table 4). The $p$ value was 0.000 and was statistically significant. The calculated $\mathrm{k}$ value was 0.659 which implies that there is no significant difference between the Muller's maneuver and DISE.

\section{Discussion}

Polysomnography is the standard diagnostic method for OSA, and it is useful for evaluating the severity of OSA. But polysomnography cannot evaluate the sites of upper airway collapse. The awake examinations of upper airway were done to visualize the site and the pattern of airway collapse in OSA patients; however, the success of this method is controversial as it is done in the awake state as there will be increased muscle tone.

Croft and Pringle ${ }^{3}$ in 1991 introduced the sleep endoscopy where the upper airway could be evaluated after attaining the druginduced sleep. The DISE can result in a sleep similar to physiological sleep; hence, the airway evaluation will be more appropriate with DISE than awake evaluation in OSA patients.

The upper airway collapse sites seen in awake and DISE are found to be variable in several studies. ${ }^{4,5}$ Few literatures have stated these variabilities for the decision of correct choice of surgery in the OSA patients. Only a very few studies found the importance of DISE for the success of surgery.

For the appropriate surgical planning in OSA, the detection of severe airway collapse in different sites is important. Severe collapse at the retrolingual level is an important risk for the failure of surgery in OSA patients. ${ }^{6}$ Therefore, it is important for the airway evaluation preoperatively in patients with OSA to show the actual severity and level of collapse during sleep which will help for the correct surgical interventions. However, the surgeries for all the collapse sites seen in DISE may not be successful, ${ }^{7}$ and DISE can show extra sites of obstruction which lead to unnecessary procedures.

In the current study, we have evaluated 30 patients with OSA who were diagnosed with OSA in polysomnography. All the study subjects in the present study showed multilevel collapse and two

Table 3: Comparison between type of airway collapse at retropalatal level

\begin{tabular}{lcc}
\hline $\begin{array}{l}\text { Type of airway } \\
\text { collapse at } \\
\text { retropalatal level }\end{array}$ & Muller's maneuver & $\begin{array}{l}\text { Drug-induced sleep } \\
\text { endoscopy }\end{array}$ \\
\hline Anteroposterior & $3(10.0 \%)$ & $4(13.4 \%)$ \\
Lateral & $11(36.7 \%)$ & $7(23.3 \%)$ \\
Circumferential & $16(53.3 \%)$ & $19(63.3 \%)$ \\
Total & $30(100.0 \%)$ & $30(100.0 \%)$ \\
\hline
\end{tabular}

Table 4: Comparison between type of airway collapse in retrolingual level

\begin{tabular}{lcc}
\hline $\begin{array}{l}\text { Type of airway } \\
\text { collapse }\end{array}$ & Muller's maneuver & $\begin{array}{l}\text { Drug-induced sleep } \\
\text { endoscopy }\end{array}$ \\
\hline Lateral & $8(26.6 \%)$ & $8(26.6 \%)$ \\
Circumferential & $22(73.3 \%)$ & $22(73.3 \%)$ \\
Total & $30(100.0 \%)$ & $30(100.0 \%)$ \\
\hline
\end{tabular}


patients showed collapse at epiglottis levels in addition to the other sites. Literatures so far studied also reported that multilevel obstruction is most common and in patients with a single-level obstruction, a velar-level obstruction is most frequent. ${ }^{8}$

\section{Retropalatal Level}

With regard to the type and degree of collapse, our study showed similar findings in both procedures. Of the total 30 patients, 5 patients showed $50-75 \%$ collapse with Muller's maneuver, in which 3 of them showed $>75 \%$ collapse with DISE. The circumferential collapse was seen in 16 patients with Muller's maneuver, of which 13 showed the same pattern with DISE.

Soares et al. ${ }^{9}$ conducted a retrospective study. At the retropalatal level, there was an incidence of severe collapse in $90.6 \%$ of patients with Muller's maneuver compared with $98.1 \%$ of patients evaluated via DISE.

\section{Retrolingual Level}

In our study, 26 patients showed $>75 \%$ collapse in Muller's maneuver and DISE. Among 4 patients showed $50-75 \%$ collapse with Muller's maneuver, 3 patients showed $>75 \%$ collapse with DISE. All the 26 patients showed $>75 \%$ collapse with Muller's maneuver showed the same finding in DISE.

Comparing the type of collapse at the retrolingual level, our study showed a similar type of collapse with both procedures. Of the 30 patients, 22 patients (90.9\%) showed a circumferential collapse in Muller's maneuver. Among them, 20 showed similar collapse in DISE.

Cavaliere et al. ${ }^{10}$ included 66 OSA patients, and the obstructing pattern was similar in both procedures $(p>0.05)$ for all sites. Yegïn et al. ${ }^{11}$ showed a significant correlation in identifying the airway obstruction at the level of oropharynx and velum with both endoscopic procedures.

\section{Conclusion}

Obstructive sleep apnea syndrome is a morbid condition affecting upper airway due to dynamic and multisegmental obstruction of the upper airway. So, it is important to carefully evaluate the upper airway to determine the site and pattern of upper airway obstruction in OSA patients in planning the most appropriate management. In our study, it was observed that the type and degree of upper airway collapse at the retropalatal and retrolingual levels in DISE were similar to that of Muller's maneuver in severe OSA patients. Hence, DISE is not necessarily a diagnostic tool in the assessment of upper airway obstruction in patients with severe OSA. However, few studies reported that DISE is an appropriate method in treatment decision in mild OSA patients. Further studies with larger sample size are necessary to validate the high correlation between Muller's maneuver and DISE.

\section{Ethical Approval}

The above study was approved by the institutional ethical committee.

\section{Informed Consent}

The informed consent was obtained from all individual participants included in this study.

\section{REFERENCES}

1. De Vito A, Carrasco Llatas M, Vanni A, et al. European position paper on drug-induced sedation endoscopy (DISE). Sleep Breath 2014;18(3):453-465. DOI: 10.1007/s11325-014-0989-6.

2. Bachar $G$, Nageris $B$, Feinmesser $R$, et al. Novel grading system for quantifying upper-airway obstruction on sleep endoscopy. Lung 2012;190(3):313-318. DOI: 10.1007/s00408-011-9367-3.

3. Croft C, Pringle M. Sleep endoscopy: a technique of assessment in snoring and obstructive sleep apnoea. Clin Otolaryngol Allied Sci 1991;16(5):504-509. DOI: 10.1111/j.1365-2273.1991.tb01050.x.

4. Friedman $M$, Ibrahim $H$, Bass L. Clinical staging for sleep-disordered breathing. Otolaryngol Head Neck Surg 2002;127(1):13-21. DOI: 10.1067/mhn.2002.126477.

5. Ravesloot M, de Vries N. One hundred consecutive patients undergoing drug-induced sleep endoscopy: results and evaluation. Laryngoscope 2011;121(12):2710-2716. DOI: 10.1002/lary.22369.

6. Salamanca F, Costantini F, Bianchi A, et al. Identification of obstructive sites and patterns in obstructive sleep apnoea syndrome by sleep endoscopy in 614 patients. Acta Otorhinolaryngol Ital 2013;33(4): 261-266.

7. Lin $\mathrm{H}$, Friedman $\mathrm{M}$, Chang $\mathrm{H}$, et al. The efficacy of multilevel surgery of the upper airway in adults with obstructive sleep apnoea/hypopnea syndrome. Laryngoscope 2008;118(5):902-908. DOI: 10.1097/ MLG.0b013e31816422ea.

8. Stuck B, Maurer J. Airway evaluation in obstructive sleep apnoea. Sleep Medicine Rev 2008;12(6):411-436. DOI: 10.1016/j.smrv.2007.08.009.

9. Soares D, Folbe A, Yoo G, et al. Drug-induced sleep endoscopy vs awake Müller's manoeuvre in the diagnosis of severe upper airway obstruction. Otolaryngol Head Neck Surg 2012;148(1):151-156. DOI: 10.1177/0194599812460505.

10. Cavaliere M, Russo F, lemma M. Awake vs drug-induced sleep endoscopy: evaluation of airway obstruction in obstructive sleep apnoea/hypopnoea syndrome. Laryngoscope 2013;123(9):2315-2318. DOI: 10.1002/lary.23881.

11. Yegïn Y, Çelik M, Kaya K, et al. Comparison of drug-induced sleep endoscopy and Müller's manoeuvre in diagnosing obstructive sleep apnoea using a VOTE classification system. Braz J Otorhinolaryngo 2017;83(4):445-450. DOI: 10.1016/j.bjorl.2016.05.009. 